Vittalle - Revista de Ciências da Saúde v. 30, n. 1 (2018) 56-62
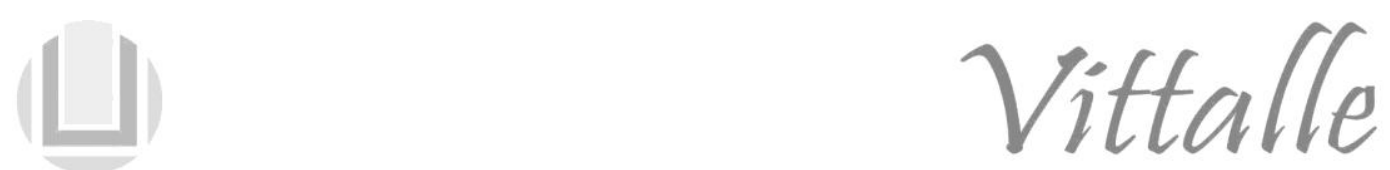

\title{
Conhecimento, uso e aceitação de acadêmicos de medicina sobre as práticas integrativas e complementares
}

\author{
Angélica Garcia Couto ${ }^{\mathrm{a}, \mathrm{b},{ }^{*}, \text { Mara Cristina Binz }}{ }^{\mathrm{c}}$, Alan De Jesus Pires De \\ Moraes $^{c}$, Bruno De Luca Serpa Caetano ${ }^{c}$, Celso Castelan Da Cunha ${ }^{c}$
}

aPrograma de Pós-graduação em Ciências Farmacêuticas, Universidade do Vale do Itajaí, Itajaí, SC, Brasil ${ }^{\mathrm{b}}$ Curso de Farmácia, Universidade do Vale do Itajaí, Itajaí, SC, Brasil

${ }^{c}$ Curso de Medicina, Universidade do Vale do Itajaí, Itajaí, SC, Brasil

Histórico do Artigo

Recebido em:

05/11/2017

Aceito em:

$30 / 03 / 2018$

Palavras-chave:

Terapias

complementares;

Sistema Único de

Saúde;

estudantes de

medicina.

Key-words

Integrative and

complementary

therapies;

Unified Health System;

students medical.

\section{RESUMO}

No Brasil, a divulgação sobre as Práticas Integrativas e Complementares (PICs) em saúde ganhou impulso na sociedade, após a sua legitimação no Sistema Único de Saúde (SUS). Entretanto, muitas dessas terapias têm encontrado resistência em sua aceitação e uso, tanto no meio acadêmico-científico, quanto por parte dos profissionais e gestores de saúde. Entre as razões, destaca-se a falta de capacitação, na fase de formação acadêmica do profissional. Dessa forma, este trabalho tem como objetivo avaliar o conhecimento, o uso e a aceitação dos acadêmicos do curso de Medicina na Universidade do Vale do Itajaí sobre as PICs. Trata-se de um estudo transversal, utilizando-se de uma pesquisa estruturada, na forma de um questionário autoaplicável, dirigido aos acadêmicos matriculados do $1^{\circ}$ ao $7^{\circ}$ período do Curso de Medicina. Dentre as práticas mais conhecidas entre os acadêmicos estão a Acupuntura, Ioga e Meditação, com mais de $90 \%$ de citação, seguida da Homeopatia e Fitoterapia com mais de $70 \%$. Fitoterapia, Meditação, Ioga, Homeopatia e Terapias Florais estão entre as mais usadas, assim como as mais aceitas, incluindo a massoterapia. Porém, o conhecimento frente à oferta das PICs pelo SUS foi de apenas $24 \%$. Esses resultados apontam para a necessidade de fortalecer as bases de formação do profissional para atuação no SUS, com uma visão ampliada, contemplando todas as possibilidades terapêuticas, às quais o usuário tem direito.

Knowledge, use and acceptance of medicine students on integrative and complementary practices

\begin{abstract}
In Brazil, the dissemination on Integrative and Complementary Practices (ICPs) in health gained momentum in society, after its legitimacy in the Unified Health System (SUS). However, many of these therapies have found resistance in their acceptance and use, both in the academic-scientific area and by professionals and health managers. Among the reasons, it is highlighted the lack of training, in the academic training of the professional. In this way, this work aims to evaluate the knowledge, use and acceptance of the medical students of a University of the Upper Vale of Itajaí on the PICs. This is a cross-sectional study, using a structured research, in the form of a self-administered questionnaire, addressed to the students enrolled from the 1 st to the 7 th period of the Medicine Course. Acupuncture, Yoga and Meditation, with more than $90 \%$ of citation, followed by Homeopathy and Phytotherapy with more than $70 \%$, are among the best known practices among academics. Phytotherapy, Meditation, Yoga, Homeopathy and Floral Therapies are among the most used. as well as the most accepted, including massage therapy. However, the knowledge about the supply of ICPs by Unified Health System (UHS)was only $24 \%$. The students' knowledge about the offer of ICPs by PSH was notoriously low, although most of them refer to their acceptance both as a user and their possible indication as a future medical professional. Only $24 \%$ of the academics knew that integrative practices are offered in the UHS. These results point to the need to strengthen the training bases of the professional to work in the UHS, with an expanded view contemplating all the therapeutic possibilities to which the user is entitled.
\end{abstract}

\footnotetext{
*Autor correspondente: angelica_gcouto@ hotmail.com (A.G. Couto)
} 


\section{Introdução}

As práticas integrativas e complementares (PICs), também chamadas alternativas, por representarem uma opção ao modelo contemporâneo e ocidental, objetivam promover equilíbrio dinâmico da energia vital do ser humano e contribuir, dessa forma, para melhorar sua relação com o meio e os outros indivíduos (1). No que se refere à abordagem terapêutica, as PICs, em sua maioria, são de base vitalista, enquanto a medicina contemporânea tem uma concepção mais mecanicista e, portanto, pouco holística, com tendência a uma excessiva instrumentalização das práticas de saúde (2).

Desde os anos de 1960, com o movimento de contracultura, tem-se observado a valorização de terapias com abordagem holística no mundo ocidental. Em apoio a essa tendência, a Organização Mundial de Saúde publicou os documentos "Estratégia de la OMS sobre Medicina Tradicional 2002-2005/2014-2023", onde preconiza a oferta de práticas integrativas e complementares nos sistemas nacionais de saúde, bem como estimula o desenvolvimento de investigações para que essa oferta seja racional, com qualidade, segurança e eficácia $(3,4)$.

No Brasil, a promulgação da Política Nacional de Práticas Integrativas e Complementares - PNPIC, em 2006, através da portaria 971 do Ministério da Saúde (MS) (5), veio contribuir para a implementação das PICs no Sistema Único de Saúde (5, 6). Inicialmente, a PNPIC reconheceu a Medicina Tradicional Chinesa, Acupuntura, Plantas Medicinais, Homeopatia e Fitoterapia, Termalismo Social/Crenoterapia. Em 2017, através da portaria 849, o MS agregou novas práticas à PNPIC: Arteterapia, Ayurveda, Biodança, Dança Circular, Meditação, Musicoterapia, Naturopatia, Osteopatia, Quiropraxia, Reflexoterapia, Reiki, Shantala, Terapia Comunitária e Integrativa e Ioga (7). Recentemente, em março de 2018, durante o primeiro Congresso Internacional de Práticas Integrativas no Brasil, o MS anunciou a aprovação da inclusão de mais 10 práticas: apiterapia, aromaterapia, bioenergética, constelação familiar, cromoterapia, geoterapia, hipnoterapia, imposição de mãos, ozonioterapia e terapia de florais (8).

Apesar da inclusão e fortalecimento das PICs no SUS, ainda não há garantia do acesso da população a essas práticas de modo efetivo. A sua implementação ainda tem encontrado resistência na aceitação e uso tanto no meio acadêmico-científico quanto por parte de profissionais e gestores de saúde. Entre as razões apontadas em literatura, citase a falta de capacitação, que depende do conhecimento apreendido nas fases de formação acadêmica do profissional (9). Nesse sentido, o presente trabalho tem por objetivo abordar o nível de conhecimento, uso e aceitação em uma população de acadêmicos de graduação do curso de Medicina da Universidade Vale do Itajaí.

\section{Materiais e Métodos}

Este estudo caracteriza-se como uma pesquisa de abordagem quantitativa, descritiva, do tipo transversal, mediante a coleta de dados primários. A população-alvo constituiuse por acadêmicos do curso de medicina de uma universidade do Alto Vale do Itajaí, cursando entre o $1^{\circ}$ e o $7^{\circ}$ período. A coleta de dados se deu ao final do primeiro semestre de 2017. O protocolo utilizado neste estudo recebeu a aprovação do Comitê de Ética em Pesquisa (CEP), da Universidade do Vale do Itajaí, com o número CAAE 67215317.3.0000.0129.

Todos os sujeitos da população alvo, maiores de 18 anos, que estavam presentes no momento da coleta de dados e aceitaram participar da pesquisa, por livre e espontânea vontade, com a assinatura do Termo de Consentimento Livre e Esclarecido (TCLE), 
foram incluídos na mesma, totalizando 108 participantes do estudo.

A obtenção dos dados foi realizada por meio de um questionário, elaborado pelos próprios autores, para ser autoaplicado, individualmente, e sem identificação do informante. Esse instrumento foi composto por questões objetivas, em duas partes. A primeira foi referente aos dados sociodemográficos que contemplaram a idade, o sexo, e o período no qual o acadêmico estava matriculado $\left(1^{\circ}\right.$ ao $7^{\circ}$ período $)$, e a segunda parte, ao conhecimento, uso e aceitação das práticas de Acupuntura, Águas Termais, Antroposofia, Aromaterapia, Cromoterapia, Fitoterapia, Terapia Floral, Homeopatia, Ioga, Massoterapia, Meditação, Musicoterapia e Reiki.

Na segunda parte, os participantes acadêmicos puderam assinalar "Sim" ou "Não" para as perguntas Você conhece?, Você acredita?, Tem interesse?, Você usa ou já usou?, Como usuário, você indicaria?, Como médico, você indicaria?, Algum profissional da saúde já lhe indicou?, Você sabia que estas PICs são oferecidas pelo SUS?". Adicionalmente, os acadêmicos puderam assinalar mais de uma opção para a pergunta Como você obteve conhecimento das PICs? (Amigos, Ensino Médio, Ensino Superior, Familiares, Internet, Jornais, Profissionais da Saúde e Televisão)".

Após a coleta, os dados obtidos foram tabulados em planilhas de Excel e categorizados para análise dos parâmetros sociodemográficos, de conhecimento, uso e aceitação das PICs. Foi aplicada a análise de correlação linear de Pearson para avaliar a relação entre alguns parâmetros (10).

\section{Resultados e Discussão}

\subsection{Características sociodemográficas dos participantes do estudo}

Dos 294 acadêmicos matriculados no $1^{\circ}$ ao $7^{\circ}$ período, $36,7 \%$ responderam ao questionário. Desses, $31 \%$ eram do $1^{\circ}$ semestre, e os demais distribuídos do $2^{\circ}$ ao $7^{\circ}$ períodos, representando, respectivamente: $14 \%, 12 \%, 12 \%, 12 \%, 10 \%$ e $14 \%$.

Em relação ao perfil sociodemográfico dos alunos, a maior prevalência foi para o sexo feminino (66,7\%), com idade entre 18 e 22 anos (78\%). A prevalência do sexo feminino entre os estudantes de medicina foi compatível com os resultados da Trajetória dos Cursos de Graduação na Saúde: 1991 a 2008 (11), e de outros dois estudos com diferentes populações, porém com o gênero feminino prevalente, sendo um deles (12) com acadêmicos de enfermagem, entre 18 e 25 anos $(63,2 \%)$, no qual o gênero feminino prevaleceu com $87,7 \%$ e outro, quanto ao uso e conhecimento por usuários da Medicina Alternativa e Complementar de Juiz de Fora (13), cuja prevalência foi de 58,8\%.

\subsection{Conhecimento, Uso e Aceitação das PICs dos participantes do estudo}

Em resposta à pergunta “Você conhece?”, 99,1\% $(\mathrm{n}=107)$ dos participantes referem ter conhecimento sobre Acupuntura, mais de $90 \%$ sobre Meditação $(\mathrm{n}=101)$ e Ioga $(\mathrm{n}=$ 102), mais de $70 \%$ sobre Homeopatia $(n=102)$ e Fitoterapia $(n=78)$, e apenas $4,6 \%(n$ = 5) para a Antroposofia. Os resultados para as demais práticas que fizeram parte do questionário estão representados na Figura 1. Na tabela 1, a frequência relativa foi expressa, considerando o total de opções assinaladas para todas as PICs.

Embora a Antroposofia seja uma racionalidade médica, chama a atenção que o seu conhecimento no meio acadêmico seja notoriamente baixo. Por outro lado, a acupuntura é uma prática da medicina tradicional oriental e revelou ser mais conhecida que a própria Fitoterapia, que é uma prática mais tradicional no Brasil que a Acupuntura.

Em 2010, resultado semelhante foi obtido no meio acadêmico com o curso de 
Enfermagem (12), apontando maior conhecimento da Acupuntura (34,4\%), frente às demais práticas: Homeopatia $(11,2 \%)$, Reiki $(7,6 \%)$, Florais $(6,2 \%)$, Ervas medicinais $(5,3 \%)$, Massoterapia $(5,3 \%)$ e Fitoterapia $(3,6 \%)$.

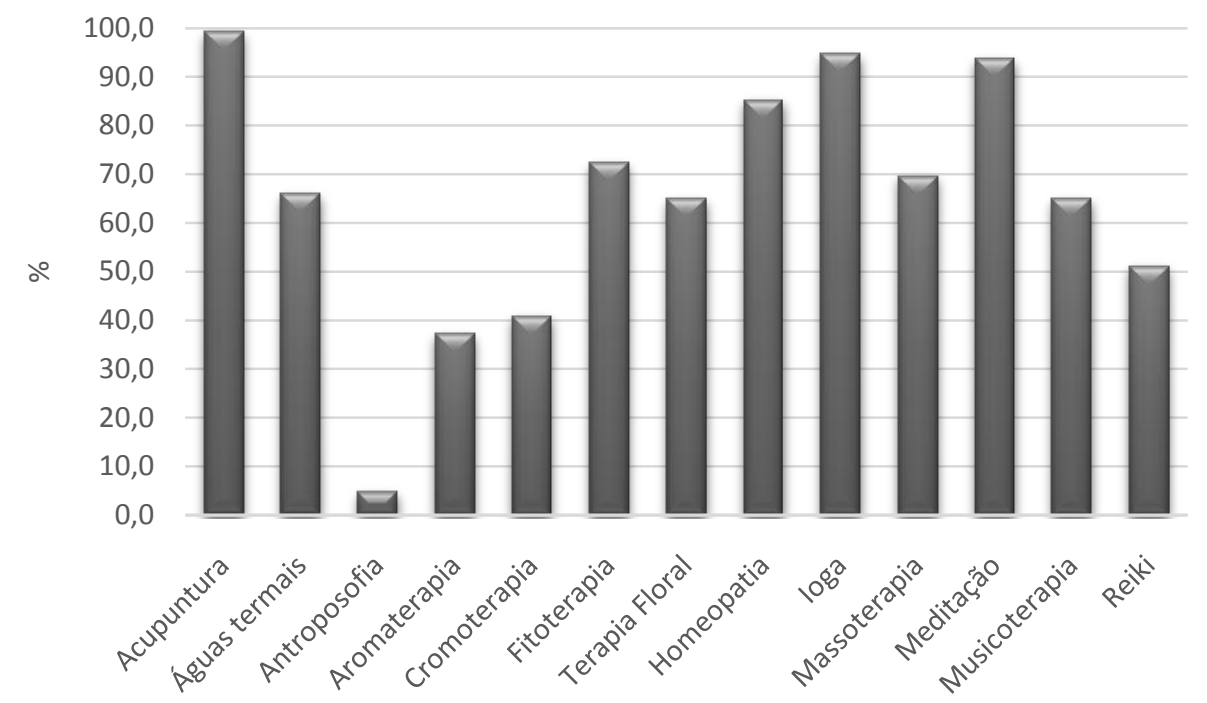

Figura 1: Percentual de conhecimento sobre as práticas integrativas pelos acadêmicos do Curso de Medicina. Universidade do Vale do Itajaí, Junho 2017.

$\mathrm{Na}$ amostra do presente estudo, o período cursado não foi discriminativo para o conhecimento das PICs pesquisadas, uma vez que a relação entre o total de respostas e o respectivo total de respondentes por período oscilou com índice de 8,5 ( $1^{\circ}$ período), 7,1 ( $3^{\circ}$ período) a 9,3 ( $5^{\circ}$ período), sugerindo que os resultados são inerentes à variabilidade amostral.

Com relação à pergunta Você acredita?, verificou-se uma redução nos índices dos que conhecem em relação aos que acreditam, de 8,5 para 5,9 no $1^{\circ}$ período, de 7,1 para 4,9 no $3^{\circ}$ período, e de 9,3 para 6,9 no $5^{\circ}$ período.

Ao serem perguntados sobre "Você tem interesse?", observou-se que a prática da Ioga foi a que mais despertou interesse, considerando o total de opções assinaladas $(n=546)$, com 13,1\% ( $\mathrm{n}=71)$, seguido da Acupuntura com 12,8\% ( $\mathrm{n}=70)$, Meditação 12,7\% (n = 69), Fitoterapia 10,2\% ( $\mathrm{n}=56)$, Massoterapia 8,7\% $(\mathrm{n}=48)$, Homeopatia e Musicoterapia, cada uma com 8,2\% $(\mathrm{n}=45)$, Reiki 6,6\% $(\mathrm{n}=36)$, Águas Termais $6,5 \%$ $(\mathrm{n}=35)$, Terapia com Florais 6,1\% $(\mathrm{n}=33)$, Cromoterapia 3,2\% $(\mathrm{n}=18)$, Aromaterapia $2,2 \%(\mathrm{n}=12)$ e Antroposofia $1,5 \%(\mathrm{n}=8)$.

No estudo de Fontanella et al. (2007) (14), sobre o interesse pelas PICs em uma comunidade da cidade de Tubarão/SC, usuária do Sistema Único de Saúde (SUS), obteve-se um alto percentual de aceitação para Fitoterapia 86,3\%, Acupuntura 84\%, Ioga 78,4\%, Homeopatia 73,8\% e Massoterapia 56,8\%.

Esse interesse pelas PICs também é salientado em outros estudos, assim como no trabalho de Greiner et al. (2000), onde foi reportado que $72 \%$ dos estudantes de medicina gostariam de ter aulas sobre terapias complementares durante o período acadêmico (15). No estudo de Hoellein et al. (2008), notou-se que $90 \%$ ou mais dos acadêmicos gostariam de aprender sobre Acupuntura e outras terapias, como Meditação e Terapia Nutricional (16).

Em resposta à pergunta "Você usa ou já usou?", verificou-se, respectivamente, por ordem de maior prevalência, o uso da Fitoterapia, Meditação, Ioga, Homeopatia e Terapias Florais, seguido das práticas de Acupuntura, Musicoterapia, Águas Termais e 
Reiki, e, em menor proporção, a Cromoterapia, Aromaterapia e Antroposofia (Tabela 1).

Esses dados corroboram os de uma pesquisa realizada em 2007, a qual levantou que $50 \%$ da população usuária do SUS, numa cidade da região Sul do Brasil, relataram fazer uso de chás, 22,7\% da Fitoterapia e Cura Espiritual, 13,6\% da Homeopatia e apenas $3,4 \%$ da Acupuntura (14).

A utilização das PICs pode ser explicada pela nova visão da medicina, na qual componentes psicológicos, emocionais e sociais fazem parte da história atual da doença (2). Schmidt et al. (2006) sugeriram que professores mais abertos ao diálogo com seus alunos influenciam-nos na busca de conhecimento científico a respeito das PICs, incentivando a pesquisa, bem como o seu uso e aceitação (17).

Ao avaliar o quesito aceitação das PICs, por meio da pergunta "Como usuário, você indicaria?", observou-se que a Fitoterapia obteve o maior índice de "Aceitação x Indicação como usuário", seguida da Meditação, Ioga e Massoterapia, Homeopatia, Acupuntura e Terapia Floral, e, em menor proporção, das demais práticas que estão relacionadas na Tabela 1.

Ainda sobre a aceitação dos entrevistados, questionou-se aos acadêmicos "Como médico, você indicaria?". Nessa perspectiva, prevaleceu a Acupuntura, seguida da Fitoterapia, Ioga e Meditação, sobre as demais práticas relacionadas na Tabela 1.

Tabela 1: Conhecimento, uso e aceitação das Práticas Integrativas e Complementares pelos acadêmicos do Curso de Medicina, sobre o total de opções assinaladas. Universidade do Vale do Itajaí, Junho 2017.

\begin{tabular}{lllll}
\hline $\begin{array}{c}\text { Práticas } \\
\text { Integrativas }\end{array}$ & \multicolumn{1}{c}{$\begin{array}{c}\text { Conhecimento } \\
(\boldsymbol{\%})\end{array}$} & Uso (\%) & $\begin{array}{c}\text { Aceitação como } \\
\text { usuário }(\mathbf{\%})\end{array}$ & $\begin{array}{c}\text { Aceitação } \\
\text { como médico } \\
(\mathbf{\%})\end{array}$ \\
\hline Acupuntura & 11,8 & 7,3 & 8,2 & 15,5 \\
Águas Termais & 7,8 & 5,8 & 5,8 & 4,7 \\
Antroposofia & 0,5 & 0,6 & 0,9 & 1,3 \\
Aromaterapia & 4,4 & 1,5 & 1,5 & 1,8 \\
Cromoterapia & 4,8 & 2,6 & 2,1 & 1,8 \\
Fitoterapia & 8,6 & 14,0 & 14,5 & 13,1 \\
Homeopatia & 10,1 & 10,8 & 9,7 & 7,8 \\
Ioga & 11,2 & 12,0 & 11,5 & 8,6 \\
Massoterapia & 8,2 & 10,5 & 10,9 & 12,9 \\
Meditação & 11,1 & 12,2 & 13,0 & 6,9 \\
Musicoterapia & 7,7 & 5,5 & 7,0 & 5,1 \\
Reiki & 6,1 & 6,1 & 6,7 & 6,8 \\
Terapia Floral & 7,7 & 11,1 & 8,2 & $\mathbf{1 0 0 , 0 0}$ \\
TOTAL & $\mathbf{1 0 0 , 0}$ & $\mathbf{1 0 0 , 0 0}$ & $\mathbf{1 0 0 , 0 0}$ & \\
\hline
\end{tabular}

Quanto ao acesso à informação sobre as PICs, obteve-se 345 respostas à pergunta Como você obteve conhecimento das PICs?, das quais $40 \%$ indicaram Amigos e Familiares ( $\mathrm{n}=72$ e 66, respectivamente), apenas 16,2\% $(\mathrm{n}=56)$ os Profissionais de saúde, o mesmo percentual a Internet $(n=56), 11,9 \%(n=41)$ o Ensino Superior (predominantemente o $1^{\circ}$ período), $4,9 \%(\mathrm{n}=17)$ o Ensino Médio, 9,3\% a TV $(\mathrm{n}=32)$ e $1,5 \%$ os Jornais $(n=5)$. Essa baixa prevalência do Profissional de saúde na origem do conhecimento sobre as PICs é um fato inerente ao histórico de formação nos cursos da área da saúde, visto que, num passado recente, a maioria das escolas médicas não provia uma educação formal sobre as PICs. Na atualidade, evidencia-se uma mudança nos quesitos acessibilidade à informação e oferta do serviço para o usuário, o que denota o fortalecimento das PICs tanto na academia quantos nos serviços de saúde (18).

As respostas à questão Algum profissional da saúde já lhe indicou? apresentaram um baixo índice de pessoas que alegaram ter recebido uma indicação profissional para o uso 
de alguma PIC. Ainda que a Fitoterapia tenha sido a prática mais indicada, compôs somente 17,4\% $(\mathrm{n}=38)$ desse perfil; sendo seguida da Acupuntura 13,3\% ( $\mathrm{n}=29)$, Homeopatia 12,4\% $(\mathrm{n}=27)$, Meditação e Terapia Floral, cada uma com $11 \%(\mathrm{n}=24)$, Ioga $10 \%(\mathrm{n}=23)$ e Massoterapia 9,6\% ( $\mathrm{n}=21)$. As demais práticas juntas contribuíram com cerca de $15 \%$ de indicação por algum profissional de saúde na amostra estudada.

Esses resultados confirmam o perfil de uso e indicações descrito por Gonçalves et al. (2008) (19), no qual 67,86\% dos médicos afirmaram nunca ter feito uso das PICs, e apenas $32,14 \%$ alegaram indicar essas práticas em sua rotina clínica no SUS. Gonçalves et al. (2008) (19) e Greiner et al. (2000) (15) ressaltam a necessidade de garantir embasamento teórico sobre PICs na formação dos profissionais, para torná-los mais aptos a avaliar a introdução ou não dessas práticas no plano terapêutico de seus pacientes.

Em conjunto, os resultados sobre o conhecimento, uso e aceitação das PICs (Tabela 1) demonstram uma forte correlação entre si, com coeficiente de correlação linear de 0,78 entre as respostas de conhecimento e uso, e de 0,75 entre as respostas de conhecimento e terapias indicadas por algum profissional da saúde. Essa análise demonstra que quanto maior o conhecimento, maior será o uso e a indicação na futura prática clínica.

Entretanto, ao se analisar o conhecimento dos acadêmicos sobre a oferta das PICs pelo SUS, observou-se que apenas $24 \%$ da amostra possuem essa informação. Desses, $50 \%$ estão concentrados no $1^{\circ}$ período, o que reflete a influência da mudança na grade curricular do Curso de Medicina, que passou a inserir as PICs como estratégia de saúde no serviço público, como parte de conteúdo da disciplina de Saúde Coletiva, no semestre em que a pesquisa foi aplicada. Somado a isso, como destacado no trabalho de Haddad et al. (11), o fato de acadêmicos de medicina pertencerem, em grande parte, a uma classe econômica mais elevada, afastariam-nos de uma rotina como usuários do SUS.

\section{Considerações finais}

Ao analisar o quesito "Conhecimento a respeito das PICs", identificou-se que o mesmo ainda é muito discreto, tanto no seu conceito e existência, como na sua oferta pelo serviço público de saúde, pois apenas $24 \%$ dos acadêmicos de Medicina sabiam que as PICs são oferecidas pelo SUS. Provavelmente, esse fato se deve à influência hegemônica da medicina convencional no sistema público de saúde, assim como a sua prevalência nas disciplinas dos cursos na área de saúde.

Mesmo com as limitações identificadas, é possível que, partindo da demanda da população e da atitude favorável dos futuros profissionais, as PICs se tornem mais conhecidas e praticadas pelos profissionais do SUS, com destaque aos profissionais da Atenção Básica à Saúde. Com isso, tais práticas podem se tornar mais respeitadas e conhecidas, menos elitizadas e mais disponíveis para toda a população brasileira, e, dessa forma, contribuírem para o fortalecimento dos princípios do SUS e da saúde da população (9).

Diante da realidade que se apresenta, é imprescindível que se fomente um amplo processo educativo, político e problematizador, que introduza as PICs na formação profissional em saúde e garanta o acesso à especialização àqueles que assim desejarem, para que se ampliem as percepções de saúde e de cuidado. Seguindo essa linha de pensamento, também é essencial que todos os cursos de formação em PICs abordem a sua proximidade com o SUS e com a saúde coletiva, para que se fortaleça a implementação das Práticas Integrativas e Complementares na saúde. 


\section{Vittalle - Revista de Ciências da Saúde v. 30, n. 1 (2018) 56-62}

\section{Agradecimentos}

Os autores agradecem à Instituição de Ensino Superior que cedeu o espaço para a realização da pesquisa, bem como aos acadêmicos que, gentilmente, cederam parte do seu tempo para contribuírem na pesquisa, tendo respondido ao questionário proposto.

Conflito de interesses: Os autores declaram que não há conflitos de interesse.

\section{Referências}

1. Telesi Júnior E. Práticas integrativas e complementares em saúde, uma nova eficácia para o SUS. Estudos Avançados 2016; 30(86): 99-112.

2. Luz MT. Cultura Contemporânea e Medicinas Alternativas: Novos Paradigmas em Saúde no Fim do Século XX. PHYSIS: Revista de Saúde Coletiva 2005; 15: 145- 176.

3. Organización Mundial de la Salud. Estrategia de la OMS sobre medicina tradicional 2002-2005. [Internet].

Disponível

em: http://apps.who.int/iris/bitstream/10665/67314/1/WHO EDM_TRM_2002.1_spa.pdf

4. Organización Mundial de la Salud. Estrategia de la OMS sobre medicina tradicional 2014-2023. [Internet]. Disponível em: http://apps.who.int/medicinedocs/documents/s21201es/s21201es.pdf

5. Brasil. Ministério da Saúde. Secretaria de Atenção à Saúde. Departamento de Atenção Básica. Política Nacional de Práticas Integrativas e Complementares no SUS - PNPIC-SUS / Ministério da Saúde, Secretaria de Atenção à Saúde, Departamento de Atenção Básica. Ministério da Saúde, 2006.

6. Brasil. Ministério da Saúde. Portaria n. 145 de 11 de janeiro de 2017. Altera procedimentos na Tabela de Procedimentos, Medicamentos, Órteses, Próteses e Materiais Especiais do SUS para atendimento na Atenção Básica. Diário Oficial da União, 2017.

7. Brasil. Ministério da Saúde. Gabinete do Ministro. Portaria no. 849 de 27 de março de 2017. Brasília, DF, 2017.

8. DAB. Notícias. [Internet]. Disponível em http://dab.saude.gov.br/portaldab/noticias.php?conteudo= \& $\&$ cod=2872

9. Azevedo E, Pelicioni MCF. Práticas integrativas e complementares de desafios para a educação. Revista Trabalho, Educação e Saúde 2012; 9: 361-378.

10. Correlação . [Internet]. http://www.inf.ufsc.br/ vera.carmo/Correlacao/Correlacao_Pearson_Spearman_Kendall.pdf

em

11. Haddad A.E, Pierantoni CR, Ristoff D, Xavier IM, Giolo J, Silva LB. A Trajetória dos Cursos de Graduação na Saúde: 1991 a 2008. Revista de Saúde Pública 2010; 44(3): 385-393.

12. Gavin ROS, Oliveira MHP, Gherardi-Donato ECS. Terapias alternativas complementares: uma visão do conhecimento dos acadêmicos de enfermagem. Ciência, Cuidado e Saúde 2010; 9(4): 760-765.

13. Neto JAC, Sirimarco MT, Neto JAD, Valle DA, Martins SJSC, Cândido TC. Uso e compreensão da medicina alternativa e complementar pela população de Juiz de Fora. Hospital Universitário Revista 2010; 36: 266-276.

14. Fontanella F, Speck FP, Piovezan AP, Kulkanp IC. Conhecimento, acesso e aceitação das práticas integrativas e complementares em saúde por uma comunidade usuária do Sistema Único de Saúde na cidade de Tubarão/SC. Arquivos Catarinenses de Medicina 2007; 36(2): 69-74.

15. Greiner KA, Murray JL, Kallail KJ. Medical student interest in alternative medicine. Journal Alternative and Complementary Medicine 2000; 6: 231-234.

16. Hoellein AR, Lineberry MJ, Kifer E. A needs assessment of complementary and alternative medicine education at the University of Kentucky College of Medicine. Medical Teacher 2008; 30 (3): 77-81.

17. Schmidt K, Rees C, Greenfield S, Wearn AM, Dennis I, Patil NG, Amri H, Boon H. Multischool, international survey of medical students' attitudes toward "Holism". Academic Medicine 2005; 80(10): 955-963.

18. Rampes H, Sharples F, Maragh S, Fisher P. Introducing complementary medicine into the medical curriculum. Journal of the Royal Society of Medicine 1997; 90: 19-22.

19. Gonçalves RP, Antunes HM, Teixeira JBP, Cardoso LO, Barbosa PR. Profissionais da área de saúde pública: atitudes, conhecimentos e experiências em relação a práticas médicas não-convencionais. Revista Atenção Primária à Saúde 2008; 11: 308-405. 